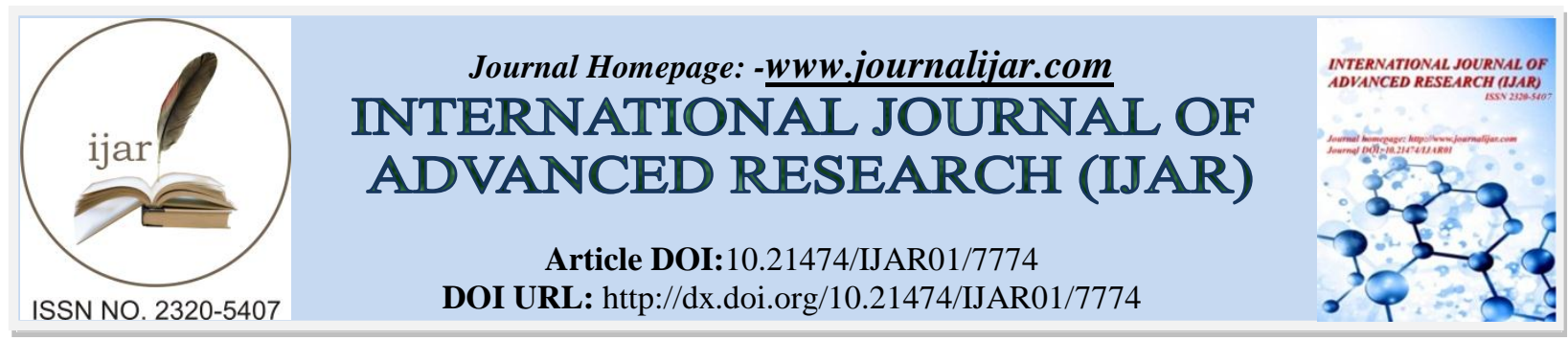

RESEARCH ARTICLE

\title{
PROFESSION INDUCED HEALTH HAZARDS OF HEALTH WORKERS IN MEDICAL PROFESSION: A SOCIOLOGICAL STUDY OF NURSES WORKING IN PRIVATE HOSPITAL OF INDIA.
}

Dr. SantoshKumar Panigrahi.

Ard Ignou, Regional Centre,Bhubaneswar.

\section{Manuscript Info}

Manuscript History

Received: 25 July 2018

Final Accepted: 31 August 2018

Published: September 2018

Keywords:-

Profession induced health

hazards, workplaceinjuries, quality of life,

coping ability, social adjustment and

psychological stress.

\begin{abstract}
Health workers in medical profession, particularly the nurses directly getaffected the physically and emotionally to various infectious diseases curbing their efficiency and quality of life. The present paperhas discussed the physical, mental and emotional health of women nurses working in corporate hospitals and its negative consequences. The paper has sought response the fundamental question that how profession induced health problems and working conditions haveadversely affected the health of women nurses and their quality of life?
\end{abstract}

Copy Right, IJAR, 2018,. All rights reserved.

\section{Introduction:-}

Health of workers in medical profession succumbed toinjuries and illness normally caused due to exposure to patients sufferings frominfectious diseases. Nurses often get affected to various diseases and injuries purely from their profession related chemical exposure and work environment.Lack of work safety environment, physical and psychological stress causes severe health problem to women nurses. Working in corporate hospitals. Excessive work pressure and major time spending for patient care (early discharge, higher patients load) which are determined at organizational level also affect health of nurses. Health status of nurses also greatly affected due to external factors such as increase use of complex technologies, innovations, numbers of very sick elderly patients (aging population). Various studies have found that health risk arising from external factors produce threat to health wellbeingof nurses and have affected their health status. Infection risk found to be eminent from very sick elderly patients (aging population) treated for terminal diseases. So organisation related risk and risk arising from external factors produce threat tonurses and affected their health status.

The very nature of work creates additional risk for nursing professional. Various health problems that the nursing community is exposed to musculoskeletal injuries, other injuries, infection, change in mental health and in the larger term, cardiovascular, metabolic and neoplastic diseases.

Long working hours \& shift work affecting health of nurses.

Hectic work schedule, like time of shift, direction and speed of rotation pattern of day off. Shift length, rest,brakes nature of job and work environment create health hazards(Barton,J et.al 1995). Shift work effect the health of women nurses as long hours may reduce the time available for sleep, leads to sleep deprivation or disturbed sleep and incomplete recovery from work. (Spark K, Fried Y et .al.,1997). Long hours of work and sleeplessness adversely affect nervous, cardiovascular,metabolic and immune functioning. This in turn effects in reducing family and social contacts which further lead to psychological stress. Long hours of work in the hospital exposes them to 
workplacehazards such as chemicals, infectious agents and physical,mental and emotional demands.Long hours of work most often compel them for smoking,alcohol consumption and other type of intoxication.

\section{Personal safety affected by night shift duty:}

Nurses on night shift and rotating duty experience long term insomnia became excessive sleeplessness make them unable to perform their normal shift duty (Drake \&Richardson,2005).Poor day time sleep and sleepless night shift are contributing factors for underperformance of many nurses. Research has found that injury risk has increased by $18 \%$ during the afternoon /evening shift and 34\% during night shift compared to morning /day shift. (Folkrd\& Tucker,2005)

\section{Social and familial disruption caused due to shift work}

Nurses working in night shiftduty onrotation basis take rest at day time which is also interrupted due to various family and household activities. Due to night shift duty they get a little time for family and friends. Night shift work posses'severe challenge for women nurses particularly with school going children and creates problem in family's social adjustment.(Preser, 2003)

\section{Negative impact of Night shift Duty}

Regular night shift duty by nurses invites gastrointestinal problem for them. Night shift duty causes sleep deprivation, effects in immune function also reported with increased risk of colon cancer. This incidence is higher in case of nurses who work three or more night shift per month for about fifteen years or more.(Schernhammer,Spizer, 2003).Frequent night shift duty and sleepless creates psychological disorders such as depression, mood swing,change of personality and other relationship difficulties.(Rohr \& Von ,2003). Various studies on occupational health of nurses revealed that the shift work increases risk for cardiovascular diseases by $40 \%$ compared to day workers(Boggild\&Knuston,1999). Studies also revealed that night shift has negative effect on reproductive health with increased risk in spontaneous abortion,preterm birth and reduced fertility in women(Frazier \& Grainger, 2003).Study reports also mentioned that night shift duty has high risk of breast cancer among women nurses, exposure to light at night reduces melatonin level, therefore increasing the risk for cancer (Megdal,Kroenke\&Lader2005). Sleep changes due to night duty in 30s and 40s mostly in younger years develop more symptoms as they grow older. Such risk include unstable angina ,myocardial infraction, hypertension ,insulin - dependentdiabetes, asthma,psychiatric illness, substance abuse, GI diseases, sleep disorders ,renal impairment , thyroid, malignant tumour and pregnancy complications. It is also increasing aging with high risk of sleeping disorders in post retirement life.

\section{Health Risk emanating from long working Hours}

Health consequence of long works duty proved fatal produces silent killer effect damagingwomen's health with significant increase in adverse physical and psychological outcome. One study has highlighted that if the working hours of shift duty exceeds more than 50 hours in a week it creates fatal consequences with multiple health problems among women nurses. Studies have also found that with increase in working hours,injuryrate increased correspondingly. Some studies have unfolded that long working hours increases the incidence of muscular, skeletal injuries and needle stick in nurses. Extended work hours and shift duty creates multiple health complication among women nurses.

\section{Diseases and injuries caused due to long hours of work and shift duty in corporate health industry}

Corporatization of health services with restructuring and redesigning of health care has led to heavy demand on nurses and other paramedical workforce.Hectic work schedule along with increased work pace and physical and psychological demands, have been reported muscular skeletal injuries and disorders(MSD)(Liscomb\& GeigerBrown 2004). MSD also varies from region to region but mostly include pain in the affected body parts for a specific duration and frequency(Bernard,1997) along with other related symptoms such as numbness and tingling. Studies have pointed out that health care workers are extremely high risk of MSD, especially for back injuries, health care workers are also overrepresented for upper extremely MSDs among workers compensation(WC) claims (Silverstein, 2002). Findings of a mega study in United States in the year 2001 revealed that nearly 108,000health workers lost their work time due to musculoskeletal injuries and disorders (Bureau of labour statistics,2002). Another path finding study in the year 2003 also revealed, the incidence rate for nonfatal occupational injuries, many of which were MSDs, was 7.9 per 100 full time equivalents (FTEs) for hospital workers(Bureau of labour and statistics,2004)Various other studies have presented a disturbing trend of nurses planned for quitting their jobs for MSD diseases related to their profession. Research in a survey of five countries found that nearly 43,000 nursing 
personnel amounting 17-39\% of nursing workforce have opted to quite their job in next year due to physical and psychological demands.(Aiken,Clarke et.al,2001). In another study nearly 6 to 11 percent of nurses reported quitting their profession due to neck shoulder and back injuries.(Trinkoff et.al,2003). Studies have revealed that MSD related injury rates for nurses have increased due to lower staffing ratios too.

\section{Professional work hazards creating physical and postural problems}

Studies have focused that majority nurses reported postural problem due to heavy lifting, bending and twisting and other manual handlings (Smedley, 1995). One study revealed that nurses were found to be at particular risk of back injuries during patient transfer, which require sudden movements in non neutral posture. Patient transfer also require flexion and rotation, increasing the injury risk due to combination of compensation, rotation and shear forces(Forde,Wegman,2002). Due to highly demanding physical work, almost all nurses developed neck shoulder and back MSD, one study observed that due to extreme flexion and frequent heavy lifting have direct impacted health of nurses with low back pain. One study has focused that operating room nurses who assists surgeons were required to maintain tension on instrument, leading to substantial musculoskeletal stress of the head and neck.As they are constantly engaged in lifting and stooping of instruments they developed arm, neck and shoulder pain. Thus constant lifting and action with arms abovesolders height develop shoulder pain and injury among working nurses.(Allen, 1990). Thus long hours of work, frequent shift retention duty affects the sleep wake cycle. This also leads to extended exposure to physical and postural risk which subsequently leads to MSD problems. One can notice a casual relation between long hours of work and incidents of musculoskeletal injuries in nurses.

\section{High Health Risk of needle sticksinjury Effecting Health Of Women Nurses}

Nurses often snecumbed to needle stick injury which is found to be highrisk exposing them to the serious and sometimes life threatening risk of blood borne infection. According to study about 600,000 to 800,000 needle stick injuries, occurs annually;(Henery,combell,2005) half of which go unreported. Nearly 1000 nurses are contracted with serious infection, such as hepatitis B or C virus or HIV from a needle stick injury. One such study also found that each year nearly 50 to 247 nurse are infected with hepatitisC virus HCV from work related needle stick (Spekowitz, Eisenbury,2005) .Since nursing staff incur most needle stick - nearly 54percentnurses get needle sticks and sharp object injuries. According to a study report about $30 \%$ nurses who receive any needle stick injury develop the risk of occupationally acquired hepatitis B virus(HBV) infection. Study findings have revealed that the risk of transmission from a positive source for hepatitis $\mathrm{c}$ is between 0.4 percent and 1.8 percentand the average risk of transmission increases with one who get injury by advice visiblycontaminated with blood and deep injury caused by needle stick.

\section{Exposure to Chemical causing health hazards}

Exposure to chemicals and other toxic substances created escalated health problem for nurses. Nurses are prone to hazards chemicalexposure in a number of ways which includes aerosols, gases, and skin contaminants- from medication used in practice. They are also prone to exposure on an acute basis. Various studies have found that substances commonly used in the health care setting can cause asthma and trigger asthma attack. Volatile organic compounds (VOCs) are chemicals that readily evaporate at room temperature, thus allowing the chemicals to be easily inhaled. The artificial spray fragrance used in the hospitals and inside the rooms create critical exposure to health workers in general and nurses in particular.(Buckley white et.al 2002) Studies have focused that most of the liquid used in spray form to keep the patient and other hospital rooms fresh but this have potential toxicthat effects human health.(Daughton, 1999). Studies also have unfolded the truth about high toxic effect of strong odours, fumes and perfumes which are potent triggers of asthma.It is always found that the hospital structures are made in such a way that it do not allow adequate indoor air circulation, thus increasing the concentration of VOCs in the air which multiplies the dose of the chemicals being inhaled.

\section{Sterilants causing health hazards of nurses.}

For sterilization purpose hospitals commonly uses ethylene oxide and glutaraldehyde, which carry high potential of causing health hazards. While cleaning equipments and work surfaces, the nurses got exposed to such chemicals .Although both of these two chemicals are powerful and effective, they are associated with serious with serious human health risk. On the otherhand glutataraldehyde is highly dangerous chemical as it is associated with respiratory irritation inhaling asthma, skin irritation and determinant and eye irritation and conjunctivitis. Nurses involved in endoscopy work are more prone to occupational asthma due to glutarldehydechemical exposure(Takingwa,2006). Besides many chemical like ETO, formaldehyde etc massively used in hospital set 
up.Studies have found that long term exposure to Eto has increased incidence to cancer causing potential, which damages the central nervous system, liver and kidneys.

\section{Medication causing Professional Health Hazards}

Health workers often confront with health problems due to medication, asantineoplasties and anaesthesia are identified as dangerous and hazardous to health of health workers. Anaesthetic gases exposed to air inhaled by workers create health problems like urinary metabolites. Studies have found that exposure to antineoplastic drugs has increased risk of spontaneous abortions among health workers.(dranitsaries,2005)

\section{Professional health hazards of nurses due to use of pesticides}

It is also Longley found that private hospitals are very frequently used pesticides both inside and outside of their facilities, which found to be serious cause of cancers for professional health hospital workers. Such application of pesticide inside the hospital building and in the periphery has special vulnerability for pregnant women. Nurses who are pregnant are highly vulnerable to pesticide exposure.

\section{Health problems created due to exposure to rubber latex}

It is Longley found that rubber latex exposure creates serious health problems for all health workers. One such study has estimated theannual incidentrate among all workers is 0.5 to 1.9 cases for 1000 full time worker per year.(Diepgen, 1999). Latex allergy start with contact dermatitis located in glove area andsymptoms can be more severe such as asthma, anaphylaxis. Continuous exposure to latex creates inability to work because of severe asthma.

\section{Inhospitable working conditions as barrier to professional health}

Researched on the said context have established that nursing work environment is intrinsically linked to health of nurses. Good work environment influences satisfaction and performance of nurses. Inhospitable work environment poses severe occupational health challenges, which include physical threat, chemical exposure and psychological stress on person as a whole.

\section{Strategic intervention and Measures to minimise occupational health hazard}

To minimise profession related health hazards of women nurses, it is quite important to take some preventive measures.Nursing as a profession may encounter severe challenges. Therefore efforts need to be initiated towards an adaptation strategy to cope with shift work and long work hours both by employers and workers. Strategies should be made in designing new work schedule and rest breaks during work, altering circadian rhythms with bright lights or blue lights,optimally timing physical activity or other work demands, improvingphysical conditioning, using caffeine,planning dietary regiments,stressreduction,support group and family counselling.(Rosa,bonnet, 1990; Monk 2000;Knauts,2003Lennernas,2004). To minimize professional health hazard of nurses Coldwell and Coldwell have suggested for use of behavioural and administrative strategies before considering pharmacologic aid. It is also suggested to cope up with shift and long hours of work, taking ashort nap by nurses could be another intervention bringing alertness. It has been also suggested by some researchers to limit the working hours to maximum 60 hours per week, which could minimise stress reflected to long working hours and shift duty.

So health problems emerging form MSD exposure through engineering controls, administrative change or workers trainings need to be for reduction of MSD risk participatory ergonomic approach to be adopted. In a participatory ergonomic approach employees participate in the identification of ergonomic risk factors, brainstormalternatives,solutions. Participatory ergonomics also has the potential for changing the culture of health care organizations, as employees begin to use ergonomic principles to improve jobs and the workplace. With participatory intervention, management commitment to reduce injuries increases, along with workers who are involved in developing solutions for positive and effective work environment.(Anoma,Steenstra\&Urlings,2003)

To deal with the problem of muscolotal injuries, a more scientific approach should be followed which implies to 1 . Patient handling, equipment, devices2. No lift policies Training on use of patient handling equipments properly. Thus to reduce MSD related risks or injuries there is need to build a culture climate of safety in terms of worker injury prevention different from patient safety culture. 
Pesticide related risk in professional health measures such as reduction of pesticide use and implementing safer integrated pest management could be minimised by initiating through a comprehensive approach that employs nontoxic and least toxic products and process that controls pest.

Measures need to be initiated to deal with latex exposure through use of low allergen power free gloves and removal of latex containing products from the worksite throughout the facility to reduce the exposure at that institution.

Measures should be taken to do away with minor and major psychological morbidity among nurses.Minor psychological morbidity that is feeling tension, anger, anxiety, depression, mental fatigue and sleep disturbance could be effectively managed through institutional involvement and counselling.

Thus, it should be mandatory for each organization to prevent assault on health care workers by restricting free moment of public in and around the hospital. To protect emergency department workers from the risk of injuries fromassault public accompanying patient.Measures should be taken for standardisation of data collection and measuring of both work environment and worker outcome.Administrative allotment for workers injury is very much needed to initiate safety measures.

\section{Conclusion:-}

In this paper we have tried to present the threat perception and health hazards of nurses caused due to risk associated to their profession. On basis of various research studies done in this topic of research certain measures are recommended to reduce health hazards of nurses associated with their profession. It can be concluded that although the health hazards and risks associated to profession and workplace are many but effective measures initiated by the organization and govt could prevent and minimize work place related injuries of nurses irrespective of government, private and corporate hospitals. Authority should initiate all possible measures to prevent work places related injuries. However the benefit of improvements to safety of nurses is of great importance as it helps retaining working nurses and new entries to the profession. All hospital management should redefine their working hours that excessively affect health of nurses which in turn also adversely impacts patients care too. To this effect as many hospitals, irrespective of government and private are making important financial investment and system level improvements to promote patients safety as well. To sum up adequate attention need to be given towards ensuring a good professional health of health workers and nurses which could given too. To accomplish a risk free health environment in the side the health facilities, the hospital management need to be careful and should promoted holistic health care environment on basis of mutual benefiting.

\section{References:-}

1. Barton J, Spelten E, Totterdell P, et al,(1995), The standard shiftwork index-a battry of questionnaires for assessingshiftwork - related problems. Work Stress. 1995;9:4-30

2. Sparks K.CooperCl,Fried Y, et al,(1997), The effects of hours of work on health: a meta-analytic review.JDccup Organ Psychol.1997;70:391-408.

3. Drake CL, Roehrs T, Richardson G, et a,(2005), Shift work sleep disorder: prevalence and consequences beyond that of symptomatic day workers. Sleep.2005;27:1453-62[PubMed]

4. Folkard S, Lombardi DA, Tucker PT,(2005),Shiftwork: safety, sleepiness and sleep.Ind Health.2005;43L20

5. [PubMed]

6. Presser $\mathrm{HB}(2003)$, Working in a 24/7 economy challenges for American families. New York:Russell Sage Foundation;2003

7. Schernhammer ES, Laden F, Speizer FE, et al(2003), Night-shift work and risk of colorectal cancer in Nurse' Health Study. J Nati Cancer 1.2003;95:825-8. [PubMed]

8. Rohr SM, Von Essen SG, Farr I.A,(2003), Overview of the Medical Consequences of Shift Work .ClinOccupEnvir Med.2003;3;351-61

9. Boggild H, KnutssonA,(1999),Shift work, risk factors and cardiovascular disease. Scand J Work Environ Health .1999;25:85-99 [PubMed]

10. Frazier LM, Grainger DA,(2003), Shift work and adverse reproductive outcomes among men and women, ClinOccup Environ Med.2003;3:279-92

11. Megdal SP, Kroenke CH, Laden F, et aI,(2005),Night work and breast cancer risk: a systematic review and meta-analysis , Rur J Cancer, 2005,41:2023-32:[PubMed]

12. Rosa RR, Bonnet MH, Bootzin RR, et al,(1990),Intervention factors for promoting adjustment to night work and shiftwork. Occup Med.1990;5:391-415[PubMed] 
13. Knauth P, HornbergerS(2003),Preventive and compensatory measures for shift workers. Occup Med (Ox) 2003;53:109-16.[PubMed].

14. LennernasM.Eating patterns. In:Becker W, editor,(2004),Project Group Chair Nordic nutrition recommendations. Copenhagen; Denmark: Norden;2004 . pp.85-101

15. Lipscomb JA>Trinkoff A, Geiger-Brown J, et al,(2004), Health care system changes ad reported musculoskeletal disorders among registered nurses. Am J of Public Health, 2004;94:1431-5. [PMC free article] [PubMed]

16. Bernard BP,(editor),(1997), Musculoskeletal disorders (MSDs) and workplace factors- a review of epidemiologic evidence for work -related musculoskeletal disorders of the neck, upper extremity and low back. Washington, DC: National Institute for Occupational Safety and Health; U.S. Government Printing Office; 1997. Publication No 97-141,

17. Kuorinka-I , Jonsson B, Kilborn A, et al,(2002) Standardized Nordic questionnaire for the analysis of musculoskeletal symptoms. Appl Ergon.1987;18:233-7. (PubMed]

18. Silverstein BE,Viikari-Juntura E, KalatJ,(2002), Use of a prevention index to identify industriesa thigh risk for work-related musculoskeltal disorders of the nect, back, and upper extremity in Washington State, 1990-1998. Am J Lnd Med, 2002;41:149-69. [PubMed]

19. Bureau of Labor and Statistics,(202) Lost-work time injuries and illnesses: characteristics and resulting time away from work,2002 U.S.Department of Labor, [Accessed December 10,2004], Publication No.04-460. Available at:http://www.bls.gov/news.release/osh2.nr0.htm

20. Bureau of Labor and Statistics,(203)Nonfatal occupational injuries and illnesses from the Survey of Occupational injuries and illnesses, 2003. [Accessed December 10,2004].Available at :http://www.bls.gov/iif/

21. Trinkoff AM, Lipscomb JA, Geiger-Brown J, et al(203) Perceived physical demand $\mathrm{s}$ and reported musculoskeletal problems in registered nurses. Am J Prev Med. 2003;24:270-5, [PubMed]

22. Smedley J, Egger P, Cooper C, et al. Manual handling activities and risk of

23. AnemaJR.Steenstra IA, Urlings IJ, et aI(2003),Participatory ergonomics as a return-to-work intervention : a future challenge? Am J Ind Med.2003;44:273-81.[PubMed \}

24. Buckley DA, Rycroft RJ, White IR, et al, Fragrance as an occupational allergen. Occup Med [London]

25. Daughton CG, Ternes TA,(1999), Pharmaceuticals and personal care products in the environment:agents of subtle change? Environ Health Perspect 1999;107:907-38 [PMC free article] [PubMed]

26. DiepgenTI.Coenraads PJ.(1999) The epidemiology of occupational contact dermatitis.IntArchOccup EnvironHealth. 1999;72:496-506 [PubMed]

27. American Nurses Association.(2005)Positionstatements.Latex allergy.2005.[Accessed November 10,2005].Available at:http://nursingworld.org/readroom/position/workplace/wkiatex.htm 